\title{
An Approximation Algorithm for MAX-2-SAT with Cardinality Constraint
}

\author{
Thomas Hofmeister \\ Informatik 2, Universität Dortmund, 44221 Dortmund, Germany \\ th01@Ls2.cs. uni-dortmund.de
}

\begin{abstract}
We present a randomized polynomial-time approximation algorithm for the MAX-2-SAT problem in the presence of an extra cardinality constraint which has an asymptotic worst-case ratio of 0.75 . This improves upon the previously best approximation ratio 0.6603 which was achieved by Bläser and Manthey [BM]. Our approach is to use a solution obtained from a linear program which we first modify greedily and to which we then apply randomized rounding. The greedy phase guarantees that the errors introduced by the randomized rounding are not too large, an approach that might be interesting for other applications as well.
\end{abstract}

\section{Introduction and Preliminaries}

In the MAXSAT problem, we are given a set of clauses. The problem is to find an assignment $a \in\{0,1\}^{n}$ to the variables $x_{1}, \ldots, x_{n}$ which satisfies as many of the clauses as possible. The MAX- $k$-SAT problem is the special case of MAXSAT where all input clauses have length at most $k$. It is already NP-hard for $k=2$, hence one has to be satisfied with approximation algorithms. An approximation algorithm for a satisfiability problem is said to have worst-case (approximation) ratio $\alpha$ if on all input instances, it computes an assignment which satisfies at least $\alpha \cdot O P T$ clauses when $O P T$ is the maximum number of clauses simultaneously satisfiable.

Approximation algorithms for MAXSAT are well-studied. On the positive side, a polynomial-time approximation algorithm is known which is based on the method of semidefinite programming and which achieves a worst-case approximation ratio of 0.7846 . For this result and an overview of the previously achieved ratios, we refer the reader to the paper by Asano and Williamson [AW]. They also present an algorithm with an approximation ratio that is conjectured to be 0.8331 .

Simpler algorithms which are based on linear programming ("LP") combined with randomized rounding achieve a worst-case ratio of 0.75 , see the original paper by Goemans and Williamson [GW1] or the books by Motwani/Raghavan ( $\mathrm{MR}$, Chapter 5.2) or Vazirani ([V], Chapter 16).

On the negative side, we mention only that Håstad [ㅍ] (Theorem 6.16) showed that a polynomial-time approximation algorithm for MAX-2-SAT with worstcase approximation ratio larger (by a constant) than $21 / 22 \approx 0.955$ would imply $\mathrm{P}=\mathrm{NP}$. 
Sometimes, it is desirable to reduce the space of feasible assignments $x \in\{0,1\}^{n}$ by extra constraints. The reason is that more problems can be transformed into such finer-grained satisfiability problems. The constraints which we consider in this paper are cardinality constraints, i.e., constraints that can be written as $x_{1}+\cdots+x_{n}=T$, where $T$ is an integer. We remark that while we consider the cardinality constraint to be an equality, other papers prefer to have an inequality " $\leq T$ " instead. It should be clear that the result obtained in our paper also extends to this alternative definition as the algorithm only needs to be applied for $T^{\prime}=0, \ldots, T$ if necessary.

Recently, cardinality-constrained variants of known NP-hard problems have obtained some attention, see e.g. SSIAS FL BM]. While Sviridenko in [S] considers the problem "MAXSATCC" which is the constrained variant of MAXSAT, Ageev and Sviridenko [AS] investigate the constrained variants of the MAXCUT and MAXCOVER problems. Feige and Langberg have shown in [FL that a semidefinite programming approach can improve the approximation ratio for some cardinality-constrained graph problems (among them the variants of MAXCUT and VERTEX COVER). The MAX-2-SATCC problem which we consider in this paper was also considered before, in the paper by Bläser and Manthey BM. Before we describe some of the results, we start with some definitions.

Definition 1. Given $n$ Boolean variables $x_{1}, \ldots, x_{n}$, an assignment to those variables is a vector $a=\left(a_{1}, \ldots, a_{n}\right) \in\{0,1\}^{n}$.

$A$ literal is either a variable $x_{i}$ or its negation $\bar{x}_{i}$. In the first case, the literal is called positive, in the second, it is called negative.

$A$ clause $C$ of length $k$ is a disjunction $C=l_{1} \vee l_{2} \vee \cdots \vee l_{k}$ of literals.

$A$ clause is called positive, if it only contains positive literals, negative, if it only contains negative literals, and pure if it is positive or negative.

A clause that is not pure will also be called mixed.

We assume in the following (without loss of generality) that each clause we are dealing with contains no variable twice, since it could be shortened otherwise.

For a fixed constant $k$, the problems MAX- $k$-SAT and MAX- $k$-SATCC ("CC" being shorthand for "cardinality constraint") are defined as follows:

Input: A set $\left\{C_{1}, \ldots, C_{m}\right\}$ of clauses each of which has length at most $k$. For the MAX- $k$-SATCC problem, an integer $T$ is also part of the input.

Problem MAX- $k$-SAT: Let $A=\{0,1\}^{n}$. Find an assignment $a \in A$ which satisfies as many of the clauses as possible.

Problem MAX-k-SATCC: Let $A=\left\{a \in\{0,1\}^{n} \mid \# a=T\right\}$, where $\# a$ denotes the number of ones in $a$. Find an assignment $a \in A$ which satisfies as many of the clauses as possible.

We note that we are considering the "unweighted" case of the problems, i.e., the input to the problems is a set of clauses and not a list of clauses.

It is well-known that already the MAX-2-SAT problem is NP-hard and since MAX-2-SAT can be solved by at most $n+1$ invocations of MAX-2-SATCC, this 
problem is NP-hard as well. Due to the negative result by Håstad mentioned above, it is also difficult to approximate beyond a ratio of $21 / 22$.

The algorithm which we describe is based on an approximation algorithm for MAXSAT by Goemans and Williamson [GW1 which uses linear programming and randomized rounding to achieve an approximation ratio 0.75 . We will later on refer to this algorithm as the "LP-based approximation algorithm". Its worstcase ratio is the same when we restrict the input to MAX-2-SAT instances.

Looking at this approximation algorithm, one might get the impression that the extra cardinality constraint does not make the problem much harder since it is easy to integrate the constraint into a linear program. Nevertheless, there is a clear hint that cardinality constraints can render satisfiability problems somewhat harder. For example, a polynomial-time algorithm for MAXSATCC with an approximation ratio larger (by a constant) than $1-(1 / e) \approx 0.632$ would mean that $\mathrm{NP} \subseteq \operatorname{DTIME}\left(n^{O(\log \log n)}\right)$, see the paper by Feige $[\mathrm{F}$, as we could approximate the SETCOVER problem to a ratio $c \cdot \ln n$ with $c<1$. This is in well-marked contrast to the fact that there are polynomial-time approximation algorithms for MAXSAT with worst-case ratio larger than 0.78.

An algorithm achieving the above-mentioned best possible ratio $1-(1 / e)$ for MAXSATCC was given in [S] where the natural question is posed whether for MAX- $k$-SATCC, $k$ fixed, better approximation ratios can be achieved.

A first answer to this question was given in [BM], where for the MAX-2-SATCC problem a polynomial-time approximation algorithm with worst-case ratio 0.6603 is described. We improve upon this result by designing a randomized polynomial-time algorithm which on input clauses $C_{1}, \ldots, C_{m}$ and input number $T$ computes an assignment $z^{\prime}$ which has exactly $T$ ones. The number $G$ of clauses that $z^{\prime}$ satisfies has the property that

$$
E[G] \geq 3 / 4 \cdot O P T_{C C}-o\left(O P T_{C C}\right),
$$

where $E[\cdot]$ denotes the expected value of a random variable and where $O P T_{C C}$ is the maximum number of clauses which can simultaneously be satisfied by an assignment with exactly $T$ ones.

With respect to the usual definitions, this means that our randomized approximation algorithm has an asymptotic worst-case ratio of $3 / 4$.

Our approach works as follows: As in the LP-based algorithm for MAXSAT, we first transform the given MAX-2-SAT instance into a linear program which can be solved in polynomial time, we only add the extra cardinality constraint to the linear program. The solution of the linear program yields $n$ parameters $y_{1}^{*}, \ldots, y_{n}^{*}$ with $0 \leq y_{i}^{*} \leq 1$ for all $i=1, \ldots, n$.

The LP-based algorithm for the general MAXSAT problem proceeds by applying randomized rounding to the $y_{i}^{*}$. On MAX-2-SAT instances, it can be shown that the so produced $\{0,1\}$-solutions on the average satisfy at least $(3 / 4) \cdot O P T$ of the clauses, where OPT is the value of the optimal MAX-2-SAT solution.

For MAX-2-SATCC, directly applying randomized rounding is prohibitive since the number of ones in the so obtained vector could be too far off the desired number $T$ of ones and correcting the number of ones by flipping some bits in the vector might change the number of satisfied clauses too much. 
Thus, our approach is to apply a technique that is called "pipage rounding" in [AS] as a preprocessing step and to then apply the normal randomized rounding to some remaining variables. We will see that the extra preprocessing step leaves us with a problem where we are better able to control the error term which is introduced by randomized rounding. The approach we use might be interesting in its own right since it shows that randomized rounding, which is an approach used in several contexts, can be improved by a greedy preprocessing phase.

\section{Linear Programming and Randomized Rounding}

We start by describing the standard approach of transforming a MAXSAT instance into a linear program which is used in the LP-based approximation algorithm.

A clause $C=l_{1} \vee \cdots \vee l_{k}$ is arithmetized by replacing negative literals $\bar{x}_{i}$ by $\left(1-x_{i}\right)$ and replacing " $\vee$ " by "+". E.g., $x_{1} \vee \bar{x}_{2}$ is transformed into $x_{1}+\left(1-x_{2}\right)$. Thus, each clause $C$ is transformed into a linear expression $\operatorname{lin}(C)$. The linear program obtained from a set of clauses $\left\{C_{1}, \ldots, C_{m}\right\}$ is as follows:

maximize $\sum_{j=1}^{m} z_{j}$

subject to $\operatorname{lin}\left(C_{j}\right) \geq z_{j}$ for all $j=1, \ldots, m$.

$$
0 \leq y_{i}, z_{j} \leq 1 \text { for all } i=1, \ldots, n, \quad j=1, \ldots, m \text {. }
$$

Assume that $z_{1}^{*}, \ldots, z_{m}^{*}, y_{1}^{*}, \ldots, y_{n}^{*}$ is the optimal solution of this linear program and that the value of the objective function on this solution is $O P T_{L P}$. Then $O P T_{L P} \geq O P T$, where $O P T$ is the maximum number of clauses simultaneously satisfiable by an assignment. The parameters $y_{1}^{*}, \ldots, y_{n}^{*}$ are used for randomized rounding:

Randomized rounding with parameters $p_{1}, \ldots, p_{n}$ randomly selects an assignment $a=\left(a_{1}, \ldots, a_{n}\right) \in\{0,1\}^{n}$ by choosing $a_{i}=1$ with probability $p_{i}$ and $a_{i}=0$ with probability $1-p_{i}$, independently for all $i=1, \ldots, n$.

For each clause $C$, there is a certain probability $P_{C}\left(p_{1}, \ldots, p_{n}\right)$ that the clause is satisfied by randomized rounding with parameters $p_{1}, \ldots, p_{n}$. It is easy to see that for every clause $C$ of length $k, P_{C}$ is a (multivariate) polynomial of degree k. E.g.:

$$
\begin{aligned}
& C=x_{1} \vee \bar{x}_{2} \quad \Rightarrow \quad P_{C}\left(p_{1}, \ldots, p_{n}\right)=1-\left(1-p_{1}\right) \cdot p_{2}=1-p_{2}+p_{1} p_{2} . \\
& C=\bar{x}_{1} \vee \bar{x}_{2} \quad \Rightarrow \quad P_{C}\left(p_{1}, \ldots, p_{n}\right)=1-p_{1} p_{2} .
\end{aligned}
$$

Note that for 0 -1-valued parameters, i.e., in the case that $p_{1}, \ldots, p_{n}$ is an assignment, $P_{C}$ yields the value 1 if the clause $C$ is satisfied and 0 otherwise. For our purposes, it is also important to note the following: If $C$ is a pure clause of length 2 , then $P_{C}\left(p_{1}, p_{2}, \ldots, p_{n}\right)$ is a polynomial in which the highest degree 
monomial has a negative coefficient -1 while for a mixed clause, the corresponding coefficient is +1 .

For a MAXSAT instance consisting of $m$ clauses $C_{1}, \ldots, C_{m}$, the following function $F$ describes the expected number of satisfied clauses if an assignment is chosen according to randomized rounding with $p_{1}, \ldots, p_{n}$.

$$
F\left(p_{1}, \ldots, p_{n}\right):=\sum_{i=1}^{m} P_{C_{i}}\left(p_{1}, \ldots, p_{n}\right)
$$

If all clauses $C_{j}$ are of length at most 2, the analysis of the LP-based MAXSAT algorithm shows that $P_{C_{j}}\left(y_{1}^{*}, \ldots, y_{n}^{*}\right) \geq 3 / 4 \cdot z_{j}^{*}$, hence

$$
F\left(y_{1}^{*}, \ldots, y_{n}^{*}\right) \geq(3 / 4) \cdot \sum_{j=1}^{m} z_{j}^{*}=(3 / 4) \cdot O P T_{L P} \geq(3 / 4) \cdot O P T
$$

A cardinality constraint $\sum_{i=1}^{n} x_{i}=T$ is a linear constraint and can easily be added to the linear program. We obtain a solution $y_{1}^{*}, \ldots, y_{n}^{*}, z_{1}^{*}, \ldots, z_{m}^{*}$ in polynomial time. Again, it holds that $F\left(y_{1}^{*}, \ldots, y_{n}^{*}\right) \geq(3 / 4) \cdot \sum_{j=1}^{m} z_{j}^{*} \geq$ $(3 / 4) \cdot O P T_{C C}$, where $O P T_{C C}$ is the maximum number of clauses which can simultaneously be satisfied by an assignment with exactly $T$ ones.

We will use the function $F$ to guide us in the search for a good assignment. The solution of the linear program gives a good enough "starting point".

Randomized rounding apparently cannot be applied directly since it can yield vectors with a number of ones that is "far away" from the desired number $T$. Repairing this by flipping some of the bits might change the $F$-value too much. Our algorithm starts with the solution $y^{*}=\left(y_{1}^{*}, \ldots, y_{n}^{*}\right)$ of the linear program (with the extra constraint) and applies a greedy preprocessing phase to the parameters. We obtain a new vector (which we still call $y^{*}$ ) and consider those positions in $y^{*}$ in more detail that are not yet 0 -1-valued. Call this set of positions $U$ : Due to the preprocessing phase, we have extra information on the mixed clauses that exist on the variables corresponding to the positions in $U$. We then show that randomized rounding performed with those variables introduces an error term which is not too large.

\section{Randomized Rounding with Preprocessing}

Our algorithm works as follows. We first transform the given set of clauses together with the cardinality constraint into a linear program, as described in the previous section. By solving the linear program, we obtain a vector $y^{*}=\left(y_{1}^{*}, \ldots, y_{n}^{*}\right) \in[0,1]^{n}$ which has the property that $F\left(y^{*}\right) \geq(3 / 4) \cdot O P T_{C C}$ and $\sum_{i=1}^{n} y_{i}^{*}=T$.

We use the vector $y^{*}$ and modify $y^{*}$ in three successive phases. First, we apply a greedy preprocessing phase where we consider pairwise positions in $y^{*}$ that are both non-integer. A similar pairwise modification has already been used in [AS] where it is named a "pipage step". Namely, in order to keep the sum of 
all $y_{i}^{*}$ unchanged, we can change two positions by increasing one of them and decreasing the other by the same amount. This can be done until one of them assumes either the value 0 or 1 . The first phase applies such changes if they increase (or leave unchanged) the value $F$.

The second phase starts if no such changes can be applied anymore. It applies randomized rounding to the remaining non-integer positions. Since this randomized rounding can produce an assignment with a number of ones which is different from $T$, we need a third, "correcting" phase.

In the description of the algorithm, we need the set of positions in $y^{*}$ that are non-integer, i.e., $U\left(y^{*}\right):=\left\{i \in\{1, \ldots, n\} \mid y_{i}^{*} \notin\{0,1\}\right\}$.

\section{Phase 1: Greedy Preprocessing}

The following two rules are applicable to pairs of positions in $U\left(y^{*}\right)$. Apply the rules in any order until none of them is applicable.

Rule 1a: If there is a pair $i \neq j$ with $i, j \in U\left(y^{*}\right)$ and $S:=y_{i}^{*}+y_{j}^{*} \leq 1$, check whether changing $\left(y_{i}^{*}, y_{j}^{*}\right)$ to $(0, S)$ or to $(S, 0)$ increases (or leaves unchanged) the $F$-value. If so, apply the change to $y^{*}$.

Rule 1b: Similar to rule $1 \mathrm{a}$, but for the case that $S:=y_{i}^{*}+y_{j}^{*}>1$. I.e., we have to check $(1, S-1)$ and $(S-1,1)$.

\section{Phase 2: Randomized rounding}

Phase 1 yields a vector $y^{*}=\left(y_{1}^{*}, \ldots, y_{n}^{*}\right) \in[0,1]^{n}$.

If $U\left(y^{*}\right)$ is empty, then the algorithm can stop with output result $:=y^{*}$.

Otherwise, we may assume for notational convenience that $U\left(y^{*}\right)=\{1, \ldots, a\}$ and that $y_{a+1}^{*}, \ldots, y_{n}^{*}$ are already $0-1$-valued. Define $s:=\sum_{i=1}^{a} y_{i}^{*}$. Since $s=$ $T-\sum_{i=a+1}^{n} y_{i}^{*}$, we know that $s$ is an integer.

Construct a vector $z \in\{0,1\}^{a}$ as follows: For $i=1, \ldots, a$, set $z_{i}:=1$ with probability $y_{i}^{*}$ and $z_{i}:=0$ with probability $1-y_{i}^{*}$, for all $i$ independently.

\section{Phase 3: Correcting}

If the number of ones in $z$ is what it should be, i.e., $\# z=s$, then this phase stops with $z^{\prime}:=z$. Otherwise, we correct $z$ as follows:

If $\# z>s$, then we arbitrarily pick $\# z-s$ positions in $z$ which we switch from one to zero to obtain a vector $z^{\prime}$ with $s$ ones.

If $\# z<s$, then we arbitrarily pick $s-\# z$ positions in $z$ which we switch from zero to one to obtain a vector $z^{\prime}$ with $s$ ones.

Finally, the algorithm outputs the assignment result $:=\left(z_{1}^{\prime}, \ldots, z_{a}^{\prime}, y_{a+1}^{*}\right.$, $\left.\ldots, y_{n}^{*}\right)$.

The number of ones in result is $T$. This is true because $\sum_{i=1}^{n} y_{i}^{*}=T$ before phase 1 . This sum is not changed by the application of the rules in phase 1 . Finally, after phase 1 and also after phases 2 and 3 , the sum $\sum_{i=1}^{a} y_{i}^{*}$ is $s$, hence result contains $s+(T-s)=T$ ones.

The running time of the algorithm is of course polynomial, since the application of a rule in phase 1 decreases $\left|U\left(y^{*}\right)\right|$, so the rules are applicable at most $n$ times. The running time is dominated by the time needed for solving the linear program. 


\section{Analyzing the Algorithm}

By the way the rules work, after phase 1 , we still have a vector $y^{*}$ with $\sum_{i=1}^{n} y_{i}^{*}=$ $T$ and $F\left(y_{1}^{*}, \ldots, y_{n}^{*}\right) \geq(3 / 4) \cdot O P T_{C C}$.

Note that since we are dealing with the MAX-2-SATCC problem, the monomials in $F$ have length at most 2 . Since phases 2 and 3 leave positions $a+1$ to $n$, i.e. $y_{a+1}^{*}, \ldots, y_{n}^{*}$, unchanged (which are 0 -1-valued), we can fix the corresponding parameters in our objective function $F$ and consider it as being dependent on the first a positions only, i.e., we can write (for some integer constants $d_{i, j}, c_{i}$ and $d)$ :

$$
\begin{aligned}
F_{a}\left(x_{1}, \ldots, x_{a}\right): & =F\left(x_{1}, \ldots, x_{a}, y_{a+1}^{*}, \ldots, y_{n}^{*}\right) \\
& =\sum_{1 \leq i<j \leq a} d_{i, j} \cdot x_{i} \cdot x_{j}+\sum_{i=1}^{a} c_{i} \cdot x_{i}+d
\end{aligned}
$$

For notational convenience (in order to avoid case distinctions), we define for arbitrary $k \neq l$ that $d_{\{k, l\}}:=d_{\min \{k, l\}, \max \{k, l\}}$.

Using simple calculus, we are now able to show that after phase 1, certain bounds on the coefficients in the remaining objective function hold:

Lemma 1. If $F_{a}\left(x_{1}, \ldots, x_{a}\right)=\sum_{1 \leq i<j \leq a} d_{i, j} \cdot x_{i} \cdot x_{j}+\sum_{i=1}^{a} c_{i} \cdot x_{i}+d$ is the objective function that we are left with after phase 1, then the following holds:

$-d \geq 0$.

$-1 \leq d_{i, j} \leq 2$ for all $1 \leq i<j \leq a$.

$-c_{i}-c_{j} \leq a$ for all $i, j \in\{1, \ldots, a\}$.

Proof. $F$ counts an expected number of satisfied clauses, which can not be negative. Hence $F_{a}(0, \ldots, 0)=d \geq 0$. For the proof of the other two properties, we will exploit the following well-known property of a real-valued function $f$ which is defined on an interval $[l, r]$ (let $f^{\prime}$ and $f^{\prime \prime}$ denote the first and second derivatives): When one of the properties a)-c) is fulfilled for all $x \in[l, r]$, then $f$ assumes its maximum on the interval $[l, r]$ at one of the endpoints of the interval:
a) $f^{\prime}(x) \leq 0$
b) $f^{\prime}(x) \geq 0$
c) $f^{\prime \prime}(x)>0$.

We know that $d_{i, j} \leq 2$, since every term $x_{i} x_{j}$ in $F$ (and thus $F_{a}$ ) is generated by a clause of length two on the variables $x_{i}$ and $x_{j}$. Only mixed clauses on $x_{i}$ and $x_{j}$ have a positive coefficient, namely +1 , on $x_{i} x_{j}$. The input clauses contain at most two mixed clauses on $x_{i}$ and $x_{j}$, since by definition, duplicate clauses in the input are not allowed. Hence, $d_{i, j} \leq 2$.

We now show that $d_{i, j}>0$ by proving that otherwise, one of the rules would be applicable. Since $d_{i, j}$ is an integer, it follows that $d_{i, j} \geq 1$.

Consider a pair $i \neq j$ of positions. The rules in phase 1 can change them while maintaining their sum $S$. In order to investigate the effect of these rules, we define the function $H(x)$ as follows: 


$$
H(x):=F_{a}(y_{1}^{*}, y_{2}^{*}, \ldots, \underbrace{x}_{\text {position } i}, \ldots, \underbrace{S-x}_{\text {position } j}, \ldots, y_{a}^{*})
$$

i.e., we fix all positions except for positions $i$ and $j$ and set the $i$-th position to $x$ and the $j$-th position in such a way that their original sum $S=y_{i}^{*}+y_{j}^{*}$ is maintained. The first and second derivatives of $H$ with respect to $x$ are:

$$
\begin{aligned}
& H^{\prime}(x)=\sum_{k \in\{1, \ldots, a\} \backslash\{i, j\}}\left(d_{\{i, k\}}-d_{\{j, k\}}\right) \cdot y_{k}^{*}+d_{\{i, j\}} \cdot S-2 \cdot d_{\{i, j\}} \cdot x+\left(c_{i}-c_{j}\right) . \\
& H^{\prime \prime}(x)=-2 \cdot d_{\{i, j\}} .
\end{aligned}
$$

When $d_{\{i, j\}}=0$, then the first derivative does not depend on $x$, hence is a constant and either a) or b) holds. When $d_{\{i, j\}}<0$, then the second derivative is larger than zero and c) is fulfilled. In both cases, $H$ assumes its maximum at one of the endpoints. Since positions $i$ and $j$ are from $U\left(y^{*}\right)$, this would mean that either rule $1 \mathrm{a}$ or rule $1 \mathrm{~b}$ would be applicable. But after phase 1 , no such rule is applicable, hence $d_{i, j}>0$ for all $i<j$.

In order to bound $c_{i}-c_{j}$, we observe that since $1 \leq d_{\{k, l\}} \leq 2$ for all $k \neq l$, we have $\sum_{k \in\{1, \ldots, a\} \backslash\{i, j\}}\left(d_{\{i, k\}}-d_{\{j, k\}}\right) \cdot y_{k}^{*} \geq-(a-2)$ as well as

$$
\begin{aligned}
d_{\{i, j\}} \cdot S-2 \cdot d_{\{i, j\}} \cdot x & =d_{\{i, j\}} \cdot(S-2 x) \\
& \geq d_{\{i, j\}} \cdot(-x) \geq-2 .
\end{aligned}
$$

This shows that $H^{\prime}(x) \geq-(a-2)-2+\left(c_{i}-c_{j}\right)=-a+\left(c_{i}-c_{j}\right)$.

If $c_{i}-c_{j}>a$, then the first derivative is larger than zero, i.e., by arguments analogous to the ones above, either rule $1 \mathrm{a}$ or rule $1 \mathrm{~b}$ would be applicable.

(We remark that $c_{i}-c_{j}$ could also be bounded in terms of $s$, but for our purposes, the above bound is enough, as we will see below.)

By renumbering the variables if necessary, we can assume w.l.o.g. that $c_{1} \geq c_{2} \geq$ $\cdots \geq c_{a}$ holds. We can rewrite the objective function as follows:

$$
F_{a}\left(x_{1}, \ldots, x_{a}\right)=\sum_{1 \leq i<j \leq a} d_{i, j} \cdot x_{i} \cdot x_{j}+\sum_{i=1}^{a}\left(c_{i}-c_{a}\right) \cdot x_{i}+\left(\sum_{i=1}^{a} x_{i}\right) \cdot c_{a}+d
$$

Let $G$ be the following function (which just omits some of the terms in $F_{a}$ ):

$$
G\left(x_{1}, \ldots, x_{a}\right):=\sum_{1 \leq i<j \leq a} d_{i, j} \cdot x_{i} \cdot x_{j}+\sum_{i=1}^{a}\left(c_{i}-c_{a}\right) \cdot x_{i} .
$$

By Lemma[1, and by the renumbering of the variables, we know that $1 \leq d_{i, j} \leq 2$ as well as $0 \leq\left(c_{i}-c_{a}\right) \leq a$. This means that $G$ is a monotone function. 
We now analyze the effect of phases 2 and 3. Define the following sets of vectors $A_{\text {int }}$ and $A_{\text {real }}$ with $A_{\text {int }} \subseteq A_{\text {real }}$ :

$$
\begin{aligned}
A_{\text {int }} & :=\left\{w \in\{0,1\}^{a} \mid \sum_{i=1}^{a} w_{i}=s\right\} \text { and } \\
A_{\text {real }} & :=\left\{w \in[0,1]^{a} \mid \sum_{i=1}^{a} w_{i}=s\right\} .
\end{aligned}
$$

At the beginning of phase 2 , we have a vector $y^{*}$ to which we apply changes in the first $a$ positions. Let $v^{*}:=\left(y_{1}^{*}, \ldots, y_{a}^{*}\right) \in A_{\text {real }}$.

From this $v^{*}$, we construct a vector $z^{\prime} \in A_{\text {int }}$. The following lemma shows that, on the average, $F_{a}\left(v^{*}\right)$ and $F_{a}\left(z^{\prime}\right)$ are not too far apart.

Lemma 2. Let $v^{*} \in A_{\text {real }}$ be given. Phases 2 and 3, when started with $v^{*}$, yield a vector $z^{\prime} \in A_{\text {int }}$ with the property that

$$
E\left[F_{a}\left(z^{\prime}\right)\right] \geq F_{a}\left(v^{*}\right)-o\left(a^{2}\right)
$$

Proof. It is clearly enough to show that

$$
E\left[G\left(z^{\prime}\right)\right] \geq G\left(v^{*}\right)-o\left(a^{2}\right)
$$

since for any vectors $w \in A_{\text {real }}, F_{a}(w)$ and $G(w)$ differ exactly by the same constant (namely $d+s \cdot c_{a}$ ).

The randomized rounding phase first computes a vector $z$. By the way the rounding is performed, we have

$$
E[\# z]=s \quad \text { as well as } E[G(z)]=G\left(v^{*}\right) .
$$

If $\# z \leq s$, then it follows that $G\left(z^{\prime}\right) \geq G(z)$. This is because $G$ is monotone and because $z^{\prime}$ is obtained from $z$ by switching zeroes to ones.

If $\# z \geq s$, then it holds that

$$
G\left(z^{\prime}\right) \geq G(z)-(\# z-s) \cdot 3 a .
$$

The reason for this is that $d_{i, j} \leq 2$ as well as $\left(c_{i}-c_{a}\right) \leq a$, hence changing a 1 to a 0 can change the $G$-value by at most $3 a$. Thus, in both cases, we can write

$$
G\left(z^{\prime}\right) \geq G(z)-|\# z-s| \cdot 3 a .
$$

By linearity of expectation, we can estimate:

$$
E\left[G\left(z^{\prime}\right)\right] \geq E[G(z)]-E[|\# z-s|] \cdot 3 a=G\left(v^{*}\right)-E[|\# z-s|] \cdot 3 a .
$$

In order to estimate $E[|\# z-s|]$, we apply Jensen's inequality (which states that "E[Y] $\leq \sqrt{\left.E\left[Y^{2}\right] "\right)}$ to $Y:=|\# z-s|$ and use $V[X]=E\left[(X-E[X])^{2}\right]$ to denote the variance of the random variable $X$. We obtain:

$$
E[|\# z-s|] \leq \sqrt{E\left[(\# z-s)^{2}\right]}=\sqrt{E\left[(\# z-E[\# z])^{2}\right]}=\sqrt{V[\# z]} .
$$


$\# z$ is the sum of independent Bernoulli-variables $z_{1}, \ldots, z_{a}$, hence $V[\# z]=$ $V\left[z_{1}\right]+\cdots+V\left[z_{a}\right]$ as well as $V\left[z_{i}\right]=\operatorname{Prob}\left(z_{i}=1\right) \cdot\left(1-\operatorname{Prob}\left(z_{i}=1\right)\right) \leq 1 / 4$, i.e., $V[\# z] \leq a / 4$ and $E[|\# z-s|] \leq \sqrt{a / 4}$.

We thus have obtained:

$$
E\left[G\left(z^{\prime}\right)\right] \geq G\left(v^{*}\right)-\sqrt{a / 4} \cdot 3 a=G\left(v^{*}\right)-o\left(a^{2}\right) .
$$

If we denote by $y^{*}$ the vector which we have arrived at after phase 1 , and result the vector which is output by the algorithm, then we have:

a) $F\left(y^{*}\right)=F\left(y_{1}^{*}, \ldots, y_{n}^{*}\right)=F_{a}\left(y_{1}^{*}, \ldots, y_{a}^{*}\right)=F_{a}\left(v^{*}\right)$.

b) $F($ result $)=F\left(z_{1}^{\prime}, \ldots, z_{a}^{\prime}, y_{a+1}^{*}, \ldots, y_{n}^{*}\right)=F_{a}\left(z_{1}^{\prime}, \ldots, z_{a}^{\prime}\right)=F_{a}\left(z^{\prime}\right)$.

c) The number of clauses satisfied by result is $F$ (result).

By c), $E[F($ result $)]$ is the number which is of interest to us, and because of b), this is equal to $E\left[F_{a}\left(z^{\prime}\right)\right]$. By Lemma 2, we have

$$
E\left[F_{a}\left(z^{\prime}\right)\right] \geq F_{a}\left(v^{*}\right)-o\left(a^{2}\right)=F\left(y^{*}\right)-o\left(a^{2}\right) \geq(3 / 4) \cdot O P T_{C C}-o\left(a^{2}\right) .
$$

It remains to be shown that the "error term" $o\left(a^{2}\right)$ is not too large compared to $O P T_{C C}$. This is what is done in the proof of the next theorem:

Theorem 1. Algorithm "Randomized Rounding with Preprocessing" is a randomized polynomial-time approximation algorithm for the MAX-2-SATCC problem with an asymptotic worst-case ratio of $3 / 4$.

Proof. Observe that the clauses $C_{1}, \ldots, C_{m}$ given as an input to the algorithm must contain at least $\left(\begin{array}{l}a \\ 2\end{array}\right)$ mixed clauses on the variables $x_{1}, \ldots, x_{a}$. The reason for this is that the objective function $F$ is the sum of the functions $P_{C}$, where $C$ ranges over the input clauses. As we have pointed out earlier, $P_{C}$ only contains a positive coefficient for $x_{i} x_{j}$ if $C$ is a mixed clause on $x_{i}$ and $x_{j}$. Since by Lemma [1. the second phase of the algorithm starts with an objective function $F_{a}$ which for all $x_{i}, x_{j} \in\left\{x_{1}, \ldots, x_{a}\right\}$ with $i \neq j$ has a coefficient $d_{\{i, j\}} \geq 1$, there must be at least $\left(\begin{array}{l}a \\ 2\end{array}\right)=\Omega\left(a^{2}\right)$ mixed clauses in the beginning. We can now prove that $O P T_{C C}=\Omega\left(a^{2}\right)$ by showing that there is an assignment with $T$ ones which satisfies $\Omega\left(a^{2}\right)$ mixed clauses.

For this purpose, we choose a vector $b=\left(b_{1}, \ldots, b_{n}\right)$ according to the uniform distribution, from the set of all assignments with exactly $T$ ones. We analyze the expected number of clauses which are satisfied by $b$ : By linearity of expectation, it is enough to compute the probability that a single (mixed) clause, say $C=x_{i} \vee \bar{x}_{j}$, is satisfied. $x_{i}$ is satisfied with probability $T / n, \bar{x}_{j}$ is satisfied with probability $(n-T) / n$. For any $T$, one of the two is at least $1 / 2$, hence $C$ is satisfied with probability at least $1 / 2$, and the expected number of clauses satisfied is at least one-half of all mixed clauses. In particular, there must be a $b$ which satisfies at least one-half of all mixed clauses. Since the given MAX-2-SATCC instance contains at least $\Omega\left(a^{2}\right)$ mixed clauses, we have that at least $\Omega\left(a^{2}\right)$ are satisfied by $b$. 
This means that $O P T_{C C}=\Omega\left(a^{2}\right)$ and for the assignment result output by the algorithm, it holds that the expected number of clauses it satisfies is $E[F($ result $)] \geq(3 / 4) \cdot O P T_{C C}-o\left(a^{2}\right) \geq(3 / 4) \cdot O P T_{C C}-o\left(O P T_{C C}\right)$.

\section{Conclusion and Outlook}

The approach which we have presented might be interesting in its own right, it could be applicable in other situations where randomized rounding is involved. Abstracting from the details, the greedy preprocessing step left us with a problem which in a certain sense is "dense", i.e., where we are left with a problem on $a$ variables, where for each pair of variables, there is one clause on those two variables in the input instance. Dense instances of problems are often easier to handle, e.g., for dense instances of the MAX- $k$-SAT problem, even polynomialtime approximation schemes (PTAS) are known, see the paper by Arora, Karger and Karpinski $\mathrm{AKK}$.

As far as MAX- $k$-SATCC for $k>2$ is concerned, the following approach might be promising: First, apply a greedy preprocessing phase which leaves a dense instance. Then, apply the techniques from AKK] for MAX- $k$-SAT to this instance. This might give approximation algorithms which have an approximation ratio better than $1-(1 / e)$ (tending to this value if $k$ gets large, of course).

The reader familiar with the article $\mathrm{AKK}$ might wonder whether the results in there could perhaps be directly applied to the MAX-2-SATCC instance, but there is a clear sign that this is not the case: As we have mentioned before, the existence of a polynomial-time approximation algorithm for MAX-2-SAT with worst-case approximation ratio larger than $21 / 22$ would imply $\mathrm{P}=\mathrm{NP}$, so an analogous statement does hold for MAX-2-SATCC. On the other hand, AKK] even yields PTASs, hence it should be clear that some sort of greedy preprocessing step is needed.

In this paper, we have not considered the weighted version of the MAX-2-SATCC problem. The reason for this is that the computations become a little bit more complicated and would obscure the main idea behind our algorithm.

Let us finish our remarks with an open problem. The MAXCUT problem, which is a special graph partitioning problem, is known to have a polynomial-time approximation algorithm with an approximation ratio of 0.878 [GW2]. Yet, its cardinality constrained variant - where the size of one of the parts is given in advance - can up to now not be approximated to a similar degree. The best known approximation ratio was achieved by Feige and Langberg [FL] who proved that with the help of semidefinite programming, an approximation ratio of $1 / 2+$ $\varepsilon$, for some $\varepsilon>0$, can be obtained. The main message behind this result is that also for cardinality-constrained problems, semidefinite programming can lead to approximation ratios which are better than those known to be achievable by linear programming. The question remains whether it is possible to apply semidefinite programming to also obtain better approximation algorithms for the MAX-2-SATCC problem. 


\section{References}

[AKK] S. Arora, D. R. Karger and M. Karpinski, Polynomial Time Approximation Schemes for Dense Instances of NP-Hard Problems, J. Computer and System Sciences 58(1), 193-210, 1999.

[AS] A. A. Ageev and M. Sviridenko, Approximation Algorithms for Maximum Coverage and Max Cut with Given Sizes of Parts, Proc. of the Seventh Conference on Integer Programming and Combinatorial Optimization (IPCO), 17-30, 1999.

[AW] T. Asano and D. P. Williamson, Improved Approximation Algorithms for MAX SAT, J. Algorithms 42(1), 173-202, 2002.

[BM] M. Bläser and B. Manthey, Improved Approximation Algorithms for MAX2SAT with Cardinality Constraints, Proc. of the Int. Symp. on Algorithms and Computation (ISAAC), 187-198, 2002.

[F] U. Feige, A Threshold of $\ln n$ for Approximating Set Cover, J. of the ACM 45(4), 634-652, 1998.

[FL] U. Feige and M. Langberg, Approximation Algorithms for Maximization Problems Arising in Graph Partitioning, J. Algorithms 41(2), 174-211, 2001.

[GW1] M. X. Goemans and D. P. Williamson, New 3/4-Approximation Algorithms for the Maximum Satisfiability Problem, SIAM J. Discrete Mathematics, 7(4), 656-666, 1994.

[GW2] M. X. Goemans and D. P. Williamson, Improved Approximation Algorithms for Maximum Cut and Satisfiability Problems Using Semidefinite Programming, J. of the ACM 42(6), 1115-1145, 1995.

[H] J. Håstad, Some Optimal Inapproximability Results, J. of the ACM 48(4), 798-859, 2001.

[MR] R. Motwani and P. Raghavan, Randomized Algorithms, Cambridge University Press, 1995.

[S] M. Sviridenko, Best Possible Approximation Algorithm for MAX SAT with Cardinality Constraint, Algorithmica 30(3), 398-405, 2001.

[V] V. V. Vazirani, Approximation Algorithms, Springer, 2001. 